\title{
Simultaneous Longitudinal and Lateral Flight Control Systems Design for Both Passive and Active Morphing TUAVs
}

\author{
Tugrul Oktay $^{1}$, Sezer Coban ${ }^{2}$ \\ ${ }^{I}$ Department of Aeronautical Engineering, Erciyes University, \\ 38039 Kayseri, Turkey \\ ${ }^{2}$ College of Aviation, Iskenderun Technical University, \\ 32100 Iskenderun, Turkey \\ oktay@erciyes.edu.tr
}

\begin{abstract}
In this article, simultaneous longitudinal and lateral flight control systems design for both passive and active morphing tactical unmanned aerial vehicles (TUAVs) is first time applied for autonomous flight performance maximization in the literature. For this purpose longitudinal and lateral dynamics modelling of TUAVs produced in Erciyes University, Faculty of Aeronautics and Astronautics, Model Aircraft Laboratory are considered in order to obtain simulation environments. Our produced TUAV is called as ZANKA-III which has weight of $50 \mathrm{~kg}$, range of around $3000 \mathrm{~km}$, endurance of around 28 hour, and ceiling altitude of around $12500 \mathrm{~m}$. Von-Karman turbulence modelling is used in order to model atmospheric turbulence during flight in both longitudinal and lateral simulation environments. A stochastic optimization method called as simultaneous perturbation stochastic approximation (i.e. SPSA) is also first time applied in order to obtain optimum dimensions of morphing parameters (i.e. extension ratios of wingspan and tailspan, assembly positions of wing and tailplane to fuselage) and optimum magnitudes of longitudinal and lateral controllers' gains (i.e. $P$, $I$ and $D$ gains) while minimizing cost index capturing terms about both longitudinal and lateral autonomous flight performances and there exists lower and upper constraints on all optimization variables in the literature.
\end{abstract}

Index Terms-Automatic control; closed loop system; control system; motion control; PID control.

\section{INTRODUCTION}

For the last 40 and 50 years Unmanned $A$ ir $V$ ehicles (UAVs) have been used for military operations as well as in civilian operations due to fact that they have several superiorities with respect to the classical manned vehicles. Some of these advantages are having economical production and low operation cost, having flexibility in configuration depending on customer's request and also not risking the pilot's life on dangerous missions. Some of related civilian UAV applications are mentioned next. In aerial agriculture such as crop monitoring and spraying, in photography such as film and video, and in coast guarding such as coastline and see-lane etc. UAVs have also been used during military

Manuscript received 8 February, 2017; accepted 20 June, 2017.

This research was funded by a grant (No. 115M603) from the The Scientific and Technological Research Council of Turkey (TUBITAK). operations. Some of related military UAV applications are mentioned next. In navy such as decoying missiles by the emission of artificial signatures and shadowing enemy fleets, in army such as reconnaissance and surveillance of enemy activity and air force such as radar system jamming and destruction and airfield base security. For many different UAV applications [1] can be visited.

The specific UAV regarded in this article is Tactical UAVs (i.e. TUAVs). TUAVs are heavier UAVs (from $50 \mathrm{~kg}$ to $1,500 \mathrm{~kg}$ ) that fly at higher altitudes (from $3000 \mathrm{~m}$ to $12000 \mathrm{~m}$ ) and are currently used primarily to support military applications. Tactical UAVs can be classified as: EN-TUAV (long endurance TUAV), LR-TUAV (long range TUAV), SR-TUAV (short range TUAV) and CR-TUAV (close range TUAV) [2]. Our TUAV is in the class of CRTUAV.

In this article a novel approach called as "combined design" is considered for optimum magnitude determination of flight control system parameters and morphing parameters. In traditional approach, a dynamic model of the any physical system will be controlled (e.g. a TUAV, a helicopter, etc.), also named as the "plant", is given a priori to the control system design engineer having no influence on this model's design. However, it is well-known reality that the physical system design problem and control system design problem are not irrelevant [3], [4]. Some negligible variations in UAV parameters may progress autonomous performance remarkably such as in [5]-[7]. The conventional sequential approach: first of all, design the plant, and second design the control system, does not give the best overall design [3], [4]. Elegantly, the system required to be controlled and the control system should be designed all together such that a given objective (i.e. cost index) is minimized, while there are hard constraints on the physical system and control system parameters. In this article this idea is pursued.

In this article not only any of longitudinal and lateral autopilot systems is considered, but also both of them are considered during flight control system design. For this purpose, optimum magnitudes of morphing parameters are determined not only considering any of longitudinal and 
lateral motion, but also considering both of them. In the literature there is well-known reality that for fixed-wing aircraft longitudinal and lateral motions are strongly uncoupled [8], [9]. In previous studies [10], [11] this reality was considered and longitudinal motion and lateral motion were examined independently. Magnitudes of fligth control systems were determined in order to minimize relevant cost index (i.e. longitudinal cost index or lateral cost index). However, the magnitudes of morphing parameters were different for independent longitudinal and lateral motion designs. Therefore, if the optimum results obtained using longitudinal motion is used, then the lateral motion do not contribute best closed-loop responses. Similarly, if the optimum results found using lateral motion is applied, then the longitudinal motion do not give best closed-loop responses. In this article this drawback is regarded, and longitudinal and lateral autopilot systems are simultaneously designed in order to obtain best magnitudes of morphing parameters. Therefore, a cost index capturing both longitudinal flight parameters and lateral flight parameters during magnitude determination of morphing parameters is created.

In this article simultaneous longitudinal and lateral flight control system design for passive and active morphing TUAVs are first time considered in the literature. This provides satisfactory closed-loop responses for both longitudinal and lateral motions. Combined design of flight control system design and morphing parameters were evaluated in previous studies of author [10], [11] independently for longitudinal and lateral motions. However, in this article during simultaneous design of longitudinal and lateral flight control system, combined design approach is also first time regarded in the literature. This ensures pleasant autonomous performance. Moreover, in this article for combined design during cost index minimization capturing terms from both longitudinal and lateral flight performance, a stochatic optimization method is first time applied in the literature. This supplies fast convergence of algoritm and obtaining safe optimum results.

\section{TUAV AND DYNAMIC MODELS OF TUAVS}

In Fig. 1 our passively and actively morphing TUAV is illustrated. HT refers horizontal tail there. Photo taken before real-time flight and also upper technical view of it are given in this figure. Due to the passive morphing property, wing and HT are able to move forward and backward in longitudinal direction independently in a prescribed interval one time before flight.

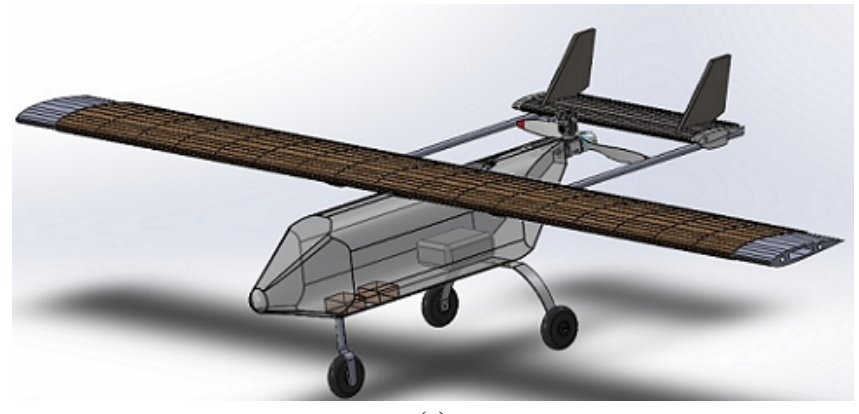

(a)

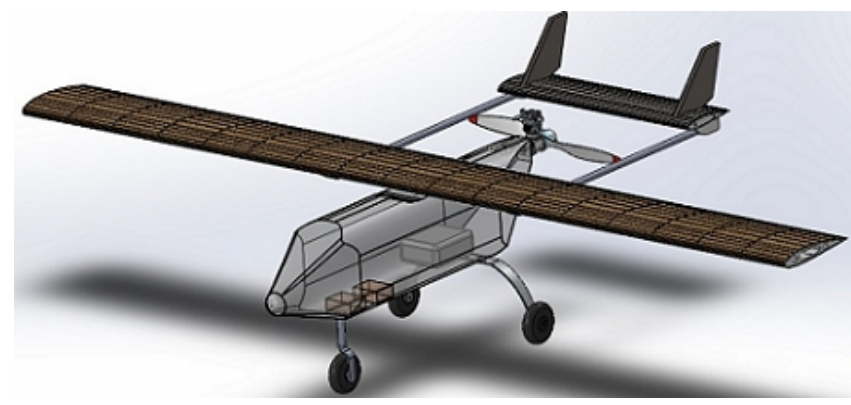

(b)

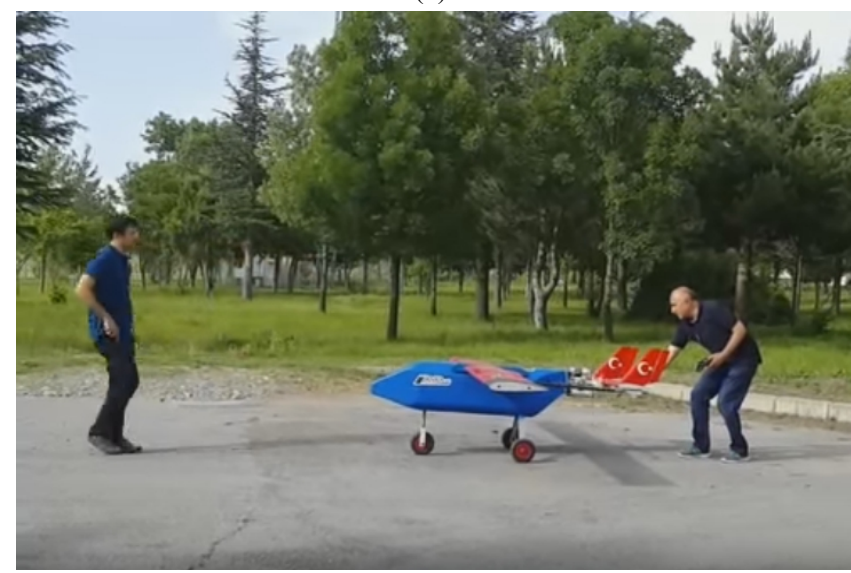

(c)

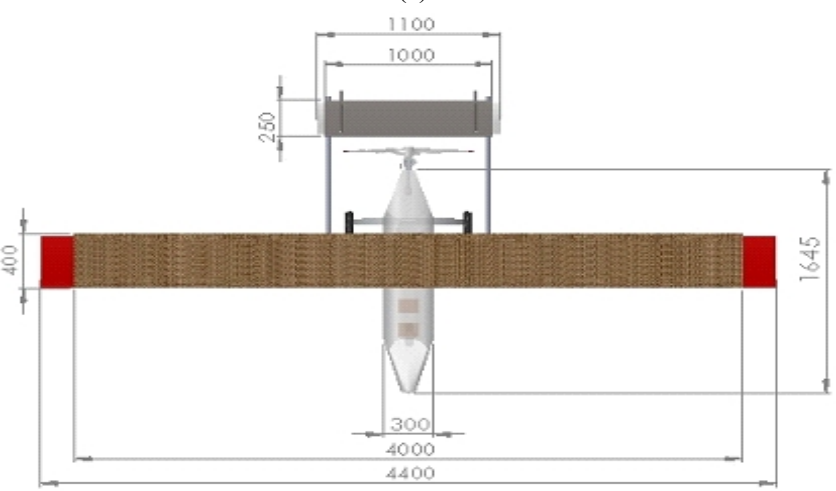

(d)

Fig. 1. Fully active + fully passive morphed wing (a) and HT TUAV; (b) no active + no passive morphed wing and HT TUAV; (c) - TUAV before flight; (d) - upper technical view of TUAV (mm s are used).

In addition, due to the active morphing property wing and HT are able to extend in spanwise direction independently in a prescribed extension interval continuously during flight.

Some technical properties of our manufactured TUAV named as ZANKA-III are given in Table I.

TABLE I. SOME PROPERTIES OF OUR TUAV.

\begin{tabular}{|c|c|}
\hline Property & Magnitude \\
\hline Total Weight & $50 \mathrm{~kg}$ \\
\hline Payload & $15 \mathrm{~kg}(5 \mathrm{~kg}$ of fuel $)$ \\
\hline Length and Width & 4 m wing span \\
& $2.188 \mathrm{~m}$ longitudinal length \\
\hline Engine Power (HP) & $18 \mathrm{Hp}$ \\
\hline Maximum Range & $2550 \mathrm{~km}$ \\
\hline Maximum Endurance & $28.7 \mathrm{~h}$ \\
\hline Speed For Maximum Endurance & $88.9 \mathrm{~km} / \mathrm{h}$ \\
\hline Ceiling Altitude & $12792 \mathrm{~m}$ \\
\hline
\end{tabular}

In (1) longitudinal and lateral state-space models for fixed-wing UAVs are given respectively. In (1) $u, w, q, \theta$, 
$\delta_{T}$ and $\delta_{e}$ refer longitudinal velocity, vertical velocity, angular velocity of pitching motion, pitch angle of UAV, throttle control and elevator control, respectively. In (2) $v, p$, $r, \phi, \delta_{a}$ and $\delta_{r}$ refer lateral velocity, angular velocity of rolling motion, angular velocity of yawing motion, roll angle of UAV, aileron control and rudder control, respectively:

$$
\begin{aligned}
& {\left[\begin{array}{c}
\Delta \dot{u} \\
\Delta \dot{w} \\
\Delta \dot{q} \\
\Delta \dot{\theta}
\end{array}\right]=\left[\begin{array}{cccc}
X_{u} & X_{w} & 0 & -g \\
Z_{u} & Z_{w} & u_{0} & 0 \\
M_{u}+M_{\dot{w}} Z_{w} & M_{w}+M_{\dot{w}} Z_{w} & M_{q}+M_{\dot{w}} u_{0} & 0 \\
0 & 0 & 1 & 0
\end{array}\right]\left[\begin{array}{c}
\Delta u \\
\Delta w \\
\Delta q \\
\Delta \theta
\end{array}\right]+\left[\begin{array}{cc}
X_{\delta_{T}} & X_{\delta_{e}} \\
Z_{\delta_{T}} & Z_{\delta_{e}} \\
M_{\delta_{T}}+M_{\dot{w}} Z_{\delta_{T}} & M_{\delta_{e}}+M_{\dot{w}} Z_{\delta_{e}} \\
0 & 0
\end{array}\right]\left[\begin{array}{l}
\Delta \delta_{T} \\
\Delta \delta_{e}
\end{array}\right],} \\
& {\left[\begin{array}{c}
\Delta \dot{v} \\
\Delta \dot{p} \\
\Delta \dot{r} \\
\Delta \dot{\phi}
\end{array}\right]=\left[\begin{array}{cccc}
Y_{v} & Y_{p} & -\left(u_{0}-Y_{r}\right) & -g \cos \left(\theta_{0}\right) \\
L_{w}^{*}+\frac{I_{x z}}{I_{x}} N_{v}^{*} & L_{p}^{*}+\frac{I_{x z}}{I_{x}} N_{p}^{*} & L_{r}^{*}+\frac{I_{x z}}{I_{x}} N_{r}^{*} & 0 \\
N_{v}^{*}+\frac{I_{x z}}{I_{z}} L_{v}^{*} & N_{p}^{*}+\frac{I_{x z}}{I_{z}} L_{p}^{*} & N_{r}^{*}+\frac{I_{x z}}{I_{z}} L_{r}^{*} & 0 \\
0 & 1 & 0 & 0
\end{array}\right]\left[\begin{array}{c}
\Delta v \\
\Delta p \\
\Delta r \\
\Delta \phi
\end{array}\right]+\left[\begin{array}{cc}
0 & Y_{\delta_{r}} \\
N_{\delta_{a}}^{*}+\frac{I_{x z}}{I_{z}} L_{\delta_{a}}^{*} & N_{\delta_{r}}^{*}+\frac{I_{x z}}{I_{z}} L_{\delta_{r}}^{*} \\
0 & 0
\end{array}\right]\left[\begin{array}{c}
I_{\delta_{a}}+\frac{I_{\delta_{a}}^{*}}{I_{x}} \\
L_{\delta_{r}}^{*}+\frac{I_{x z}}{I_{x}} N_{\delta_{r}}^{*} \\
\Delta \delta_{r}
\end{array}\right] .}
\end{aligned}
$$

Due to the passive and active morphing, stability derivatives change. For example, when any morphing does not occur, one of longitudinal derivative $X_{u}$ is

$$
X_{u}=\frac{\left(-C_{D_{u}}-2 C_{D_{0}}+C_{T_{u}}\right) Q S}{m u_{0}} .
$$

Above $S$ is the wing area and therefore after active morphing, $X_{u}$ changes and it is

$$
X_{u}=\frac{\left(-C_{D_{u}}-2 C_{D_{0}}+C_{T_{u}}\right) Q S\left(1+M_{w}\right)}{m u_{0}},
$$

where $M_{w}$ is the morphing ratio for wing. Morphing also affects magnitudes of inertial parameters and some other parameters. In our application, maximum allowable $M_{w}$ is equal to $10 \%$.

\section{Flight CONTROL SySTEM}

Structure of our autopilot system used for autonomous flight of ZANKA-III is summarized next. Hardware of our typical off-the-shelf autopilot system includes sensor packages (i.e. GPS receiver, accelerometer, magnetometer, gyro, pitot-tube) in order to obtain states for control. Software of our autopilot system consists of state estimator (i.e. Kalman filter) and path-following controller. Typical off-the-shelf autopilot system can be divided into three different layers: 1) the inner loop for stabilization of roll and pitch attitudes, 2) the middle loop for stabilization of heading and altitude, 3 ) the outer loop for tracking of $\mathrm{x}$ - and $y$ - positions of the UAV. The general structure of PID-based our autopilot system can be found in [12], [13]. It uses three layers PID controller to accomplish waypoint navigation.

\section{OPTIMIZATION AND SimUltaneOUS DESIGN}

Since there is complex dependence between total autonomous flight performance cost index and the constraints on the optimization variables ( $3 P-I-D$ gains for longitudinal controller, $3 P-I-D$ gains for lateral controller, and 2 passive morphing parameters of TUAV and 2 active morphing parameters of TUAV, entire of 10 parameters), computation of cost function derivatives with respect to these parameters is not analytically possible. This advocates the invitation of certain stochastic optimization techniques. In order to solve this specific problem a stochastic optimization method named as SPSA (i.e. simultaneous perturbation stochastic approximation) is applied. It was successfully benefited in similar highly-complex, constrained optimization problems before [14], [18]. SPSA has many advantages with respect to the other present methods in the literature. First of all, it is low cost since it applies only two evaluations of the objective in order to estimate the gradient [19]. In addition to previous advantage, it is also effective in solving constrained optimization problems [14]-[18].

In this article SPSA is used for simultaneous longitudinal and lateral flight control system design for passive and active morphing TUAVs This satisfies fast convergence of algoritm and obtaining safe optimum results. During this design problem, the optimum results improve both longitudinal and lateral flight performances. The cost index is given next

$$
\boldsymbol{J}=T_{r t}+T_{s t}+O S
$$

It consists of rise time, settling time and overshoot, respectively during reference trajectory tracking. For longitudinal and lateral flights the cost indexes are given respectively next:

$$
\left\{\begin{array}{l}
\boldsymbol{J}_{\text {long }}=T_{r_{\text {long }}}+T_{\text {st long }}+O S_{\text {long }} \\
\boldsymbol{J}_{\text {lat }}=T_{r_{\text {lat }}}+T_{\text {st lat }}+O S_{\text {lat }} .
\end{array}\right.
$$

Since in our application the cost index for longitudinal flight is much larger than the one for lateral flight, for the total cost index lateral cost index is multiplied with 100

$$
\boldsymbol{J}_{\text {tot }}=\boldsymbol{J}_{\text {long }}+100 * \boldsymbol{J}_{\text {lat }} \text {. }
$$

\section{Closed LoOp RESPONSES}

Simultaneous longitudinal and lateral flight control system 
design for passive and active morphing TUAVs are considered. In our application longitudinal autopilot is tracking 5 degrees of pitch angle and lateral autopilot is tracking 5 degrees of roll angle. The cost index consists of longitudinal flight terms and lateral flight terms. Combined design of flight control system design and morphing parameters is followed. At the end due to the using combined approach rather than conventional sequential approach, more cost index minimization is obtained. Moreover, since longitudinal and lateral flight control systems are designed simultaneously while determining optimum magnitudes of morphing parameters, the resulted paramaters improved both longitudinal and lateral flight. In Fig. 2 total cost improvement, relative total cost improvement, variations of longitudinal PID parameters, variations of lateral PID parameters, variations of passive morphing parameters, and variations of active morphing parameters are given respectively. In this figure $\mathrm{w}$ refers wing. The relative total energy save with respect to the default initial case (i.e. $P=50, I=5, D=50$ for both longitudinal and lateral PID controllers, $50 \%$ spanwise extension for both wing and HT, and initial longitudinal positions of wing and HT) is around $46 \%$ after applying simultaneous longitudinal and lateral flight control system design. In Fig. 2 for the first step of iteration, the number " 0 " is used.

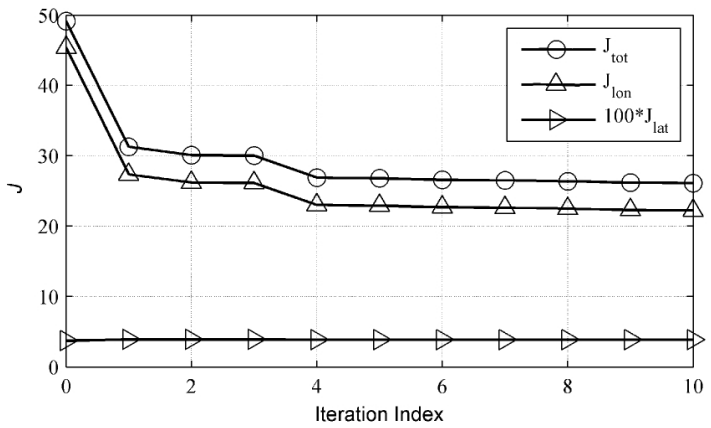

(a)

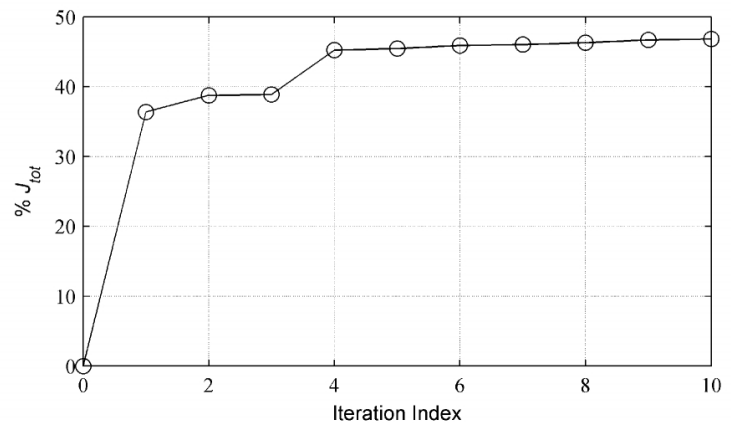

(b)

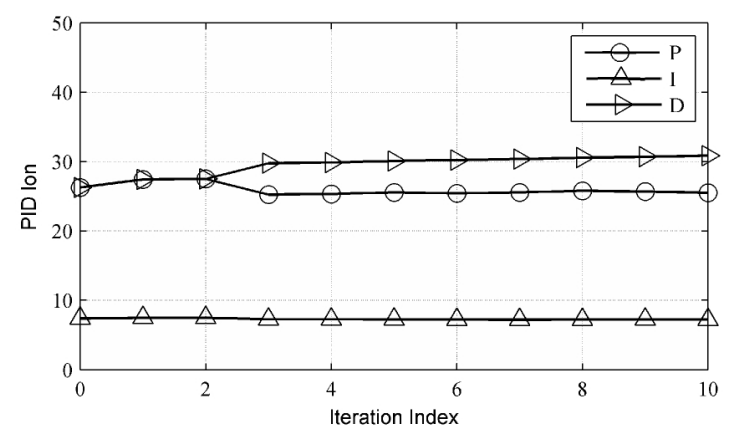

(c)

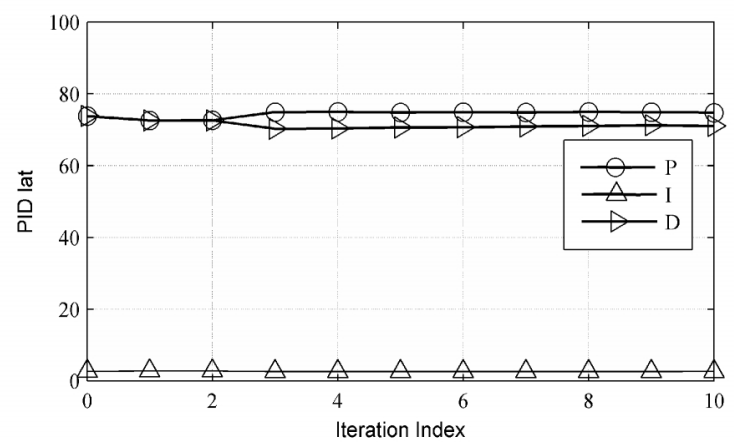

(d)

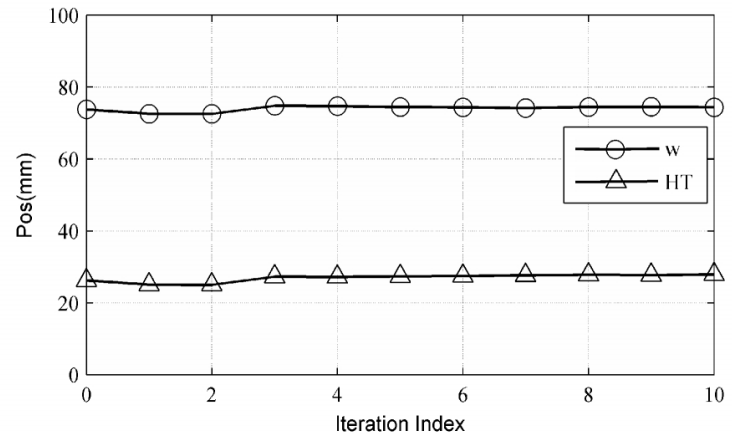

(e)

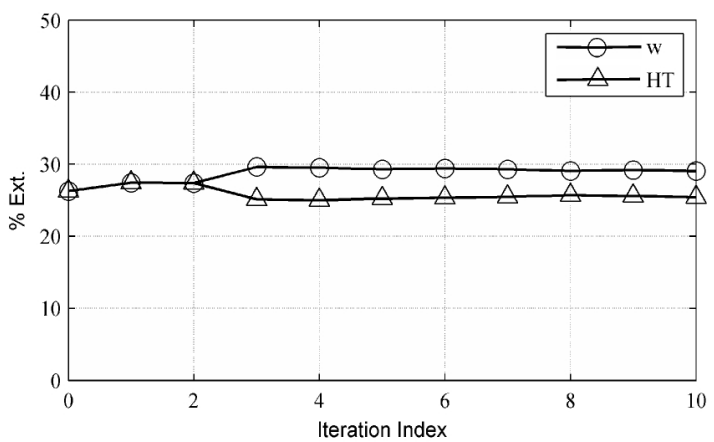

(f)

Fig 2. Cost improvement (a); relative cost improvement (b); longitudinal PID (c); lateral PID (d); passive morphing parameters (e); active morphing parameters (f).

During this design longitudinal cost is also minimized considerably. During also this design, lateral cost is not affected considerably. This result demonstrates that our simultaneous design idea do not break lateral performance while improving longitudinal performance.

Closed loop responses for both longitudinal and lateral flight while there exist atmospheric turbulance [20] is also investigated and the results obtained are given in Fig. 3 and Fig. 4 respectively. It should be noted that there is 30 and 10 degrees saturations on active surfaces, i.e. elevator for longitudinal flight and aileron for lateral flight, respectively. It can be seen from Fig. 3 and Fig. 4 that the longitudinal and lateral autopilots track desired reference trajectories succesfully. The active control surfaces also obey the constraints on them. The other outputs such as linear and angular velocities do not experience catastrophic behaviour.

\section{CONCLUSIONS}

Simultaneous longitudinal and lateral flight control systems design for both passive and active morphing tactical $u$ nmanned aerial vehicles (TUAVs) was benefited for total autonomous flight performance maximization in this article. 


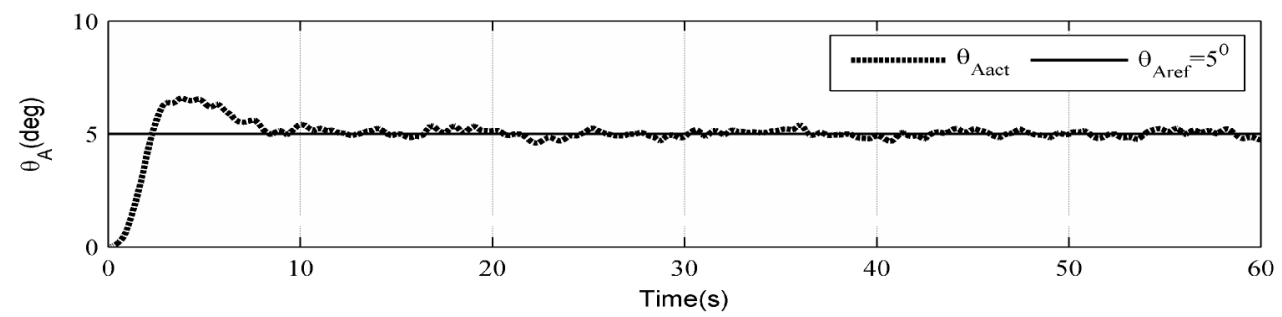

(a)

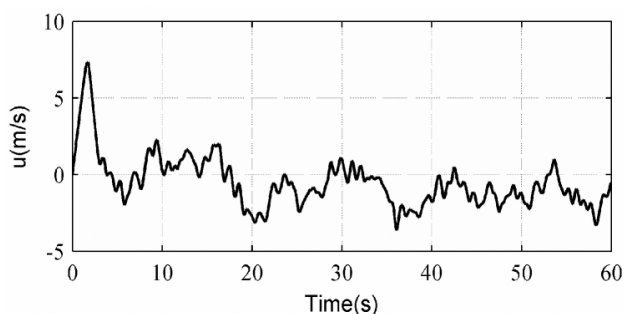

(b)

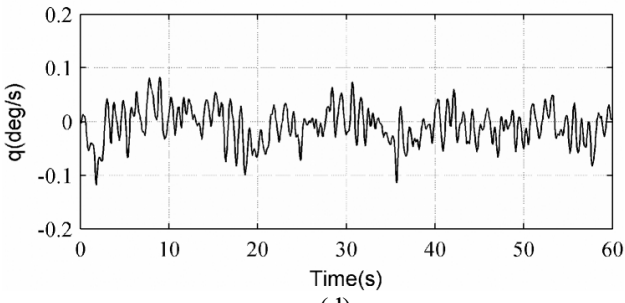

(d)

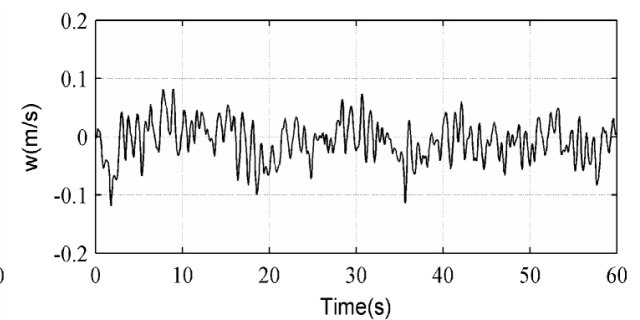

(c)

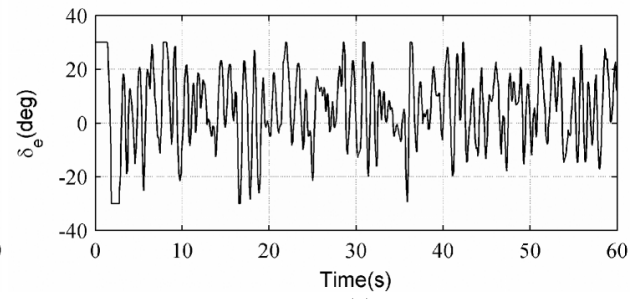

(e)

Fig. 3. Closed loop responses of longitudinal motion.

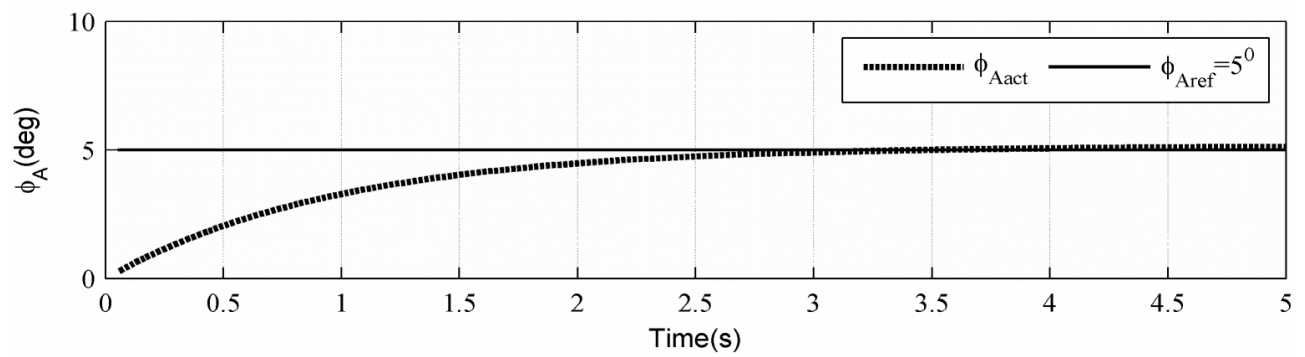

(a)

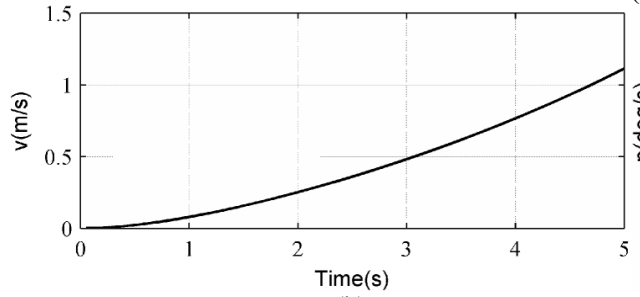

(b)

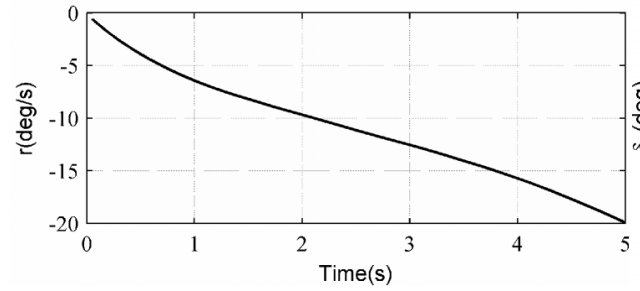

(d)

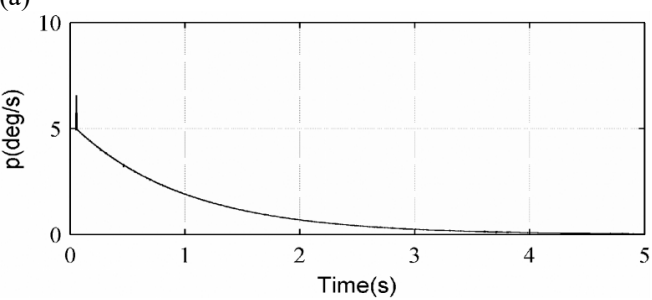

(c)

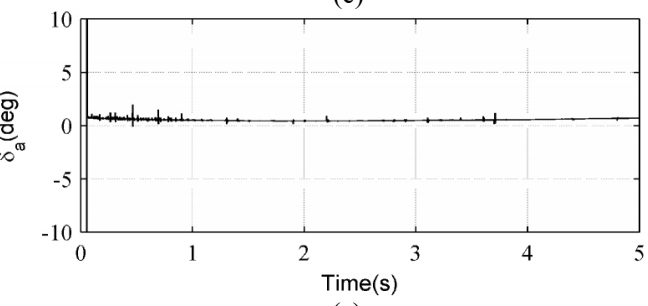

(e)

Fig. 4. Closed loop responses of lateral motion.

For this intention longitudinal and lateral dynamics modelling of TUAVs produced in Erciyes University, Faculty of Aeronautics and Astronautics, Model Aircraft Laboratory were used in order to get simulation environments. Our produced TUAV was called as ZANKAIII having weight of $50 \mathrm{~kg}$, range of around $3000 \mathrm{~km}$, endurance of around 28 hour, and ceiling altitude of around
$12500 \mathrm{~m}$. Von-Karman turbulence modelling was applied in order to model atmospheric turbulence during flight in both longitudinal and lateral simulation environments. A stochastic optimization method named as simultaneous perturbation stochastic approximation (i.e. SPSA) was used in order to obtain optimum dimensions of morphing parameters (i.e. extension ratios of wingspan and tailspan, 
assembly positions of wing and tailplane to fuselage) and optimum magnitudes of longitudinal and lateral controllers' gains (i.e. P, I and D gains) while minimizing cost index capturing terms related both longitudinal and lateral autonomous flight performances and while there were lower and upper constraints on all optimization variables.

Using SPSA, " $46 \%$ of the total cost index with respect to the default initial situation was saved." The relative total energy save was with respect to the default initial case (i.e. $P=50, I=5, D=50$ for both longitudinal and lateral PID controllers, $50 \%$ spanwise extension for both wing and HT, and initial longitudinal positions of wing and HT). Since the total cost index captures terms both related with longitudinal and lateral flights, considerable improvement in longitudinal autonomous flight performance was obtained and the lateral autonomous flight performance were not broken. Closed loop responses for both longitudinal and lateral flight while there exist atmospheric turbulance were investigated. The desired trajectories (i.e. 5 degrees pitch angle for longitudinal autopilot and 5 degrees roll angle for lateral autopilot) were successfully tracked. The saturations on active control surfaces (i.e. elevator and aileron) were also satisfied. In addition, the other outputs such as linear (i.e. $u$, $v, w)$ and angular velocities $(p, q, r)$ were not experienced with catastrophic behaviour. Simultaneous design idea converted our TUAV and its autopilot system into suitable form satisfying good performance and trajectory tracking for both longitudinal and lateral flights.

\section{REFERENCES}

[1] R. Austin, Unmanned aircraft systems. Wiley, 2010.

[2] M. F. Bento, Unmanned aerial vehicles: An Overview. Working papers, 2008. [Online]. Available: https://doi.org/10.1002/ 9780470664797

[3] K. M. Grigoriadis, M. J. Carpenter, G. Zhu, R. E. Skelton, “Optimal redesign of linear systems", in Proc. of American Control Conference, San Francisco, CA, 1993.

[4] K. M. Grigoriadis, G. Zhu, R. E. Skelton, "Optimal redesign of linear systems", Journal of Dynamic Systems, Measurement and Control, vol. 118, no. 3, pp. 598-605, 1996. [Online]. Available: https://doi.org/10.1115/1.2801186

[5] K. Park, J. W. Han, H. J. Lim, B. S. Kim, J. Lee, "Optimal design of airfoil with high aspect ratio in unmanned aerial vehicles", World Academy of Science, Engineering and Technology, vol. 2, no. 4, pp. 171-177, 2008. [Online]. Available: http://dx.doi.org/scholar. waset.org/1999.8/11529

[6] L. Krog, A. Tucker, M. Kemp, R. Boyd, "Topology optimization of aircraft wing box ribs", in Proc. of $10^{\text {th }}$ AIAA/ISSMO Multidisciplinary Analysis and Optimization Conf., Albany, New York, USA, 2004. [Online]. Available: https://doi.org/10.2514/6. 2004-4481

[7] W. Qun, J. Hong-Quang, “Optimal design of UAV's pod shape”, presented at International Symposium on Photoelectronic Detection and Imaging: Advances in Infrared Imaging and Applications, Beijing, China, 2011.

[8] R. C. Nelson, Flight Stability and Automatic Control. $2^{\text {nd }}$ ed., McGraw-Hill: New York, 2007, ch. 2-6.

[9] B. Etkin, L. D. Reid, Dynamics of Flight: Stability and Control. $3^{\text {rd }}$ ed., Hamilton Printing Company, Canada, 1996.

[10] T. Oktay, "Performance maximization of tactical unmanned aerial vehicles via integrated passive and active morphing", in 5th Int. Conf. Science, Technology, Dubai, BAE, 2016.

[11] T. Oktay, S. Coban, "Lateral autonomous performance maximization of tactical unmanned aerial vehicles by integrated passive and active Morphing", in Proc. of 6th Int. Research Conf. Science, Management and Engineering, Dubai, BAE, 2017. [Online]. Available: https://doi.org/10.24178/ijare.2017.3.1.01

[12] H. Chao, Y. Cao, Y. Q. Chen, "Autopilots for small fixed-wing unmanned aerial vehicles: A Survey”, in Proc. of IEEE Int. Conf. Mechatronics and Automation, Harbin, China, 2007.

[13] J. S. Jang, D. Liccardo, "Automation of small UAVs using a low cost mems sensor and embedded computing platform", in Proc. of $25^{\text {th }}$ Digital Avionics Systems Conf., pp. 1-9, 2006. [Online]. Available: https://doi.org/10.1109/DASC.2006.313772

[14] C. Sultan, "Proportional damping approximation using the energy gain and simultaneous perturbation stochastic approximation", Mechanical Systems and Signal Processing, vol. 24, pp. 2210-2224, 2010. [Online]. Available: http://dx.doi.org/10.1016/j.ymssp. 2010.02.013

[15] T. Oktay, Constrained control of complex helicopter models. PhD Dissertation, Virginia Tech, 2012.

[16] T. Oktay, C. Sultan, "Simultaneous helicopter and control-system design", Journal of Aircraft, vol. 50, no. 3, pp. 32-47, 2013. [Online]. Available: http://dx.doi.org/10.2514/1.C032043

[17] T. Oktay, F. Sal, "Combined passive and active helicopter main rotor morphing for helicopter energy save", Journal of the Brazilian Society of Mechanical Sciences and Engineering (BMSE), vol. 38, no. 6, pp. 1511-1525, 2016. [Online]. Available: http://dx.doi.org/ 10.1007/s40430-015-0401-x

[18] T. Oktay, M. Konar, M. Onay, M. Aydin, M. A. Mohamed, "Simultaneous small Uav and autopilot system design", Aircraft Engineering and Aerospace Technology, vol. 88, no. 6, pp. 818-834, 2016. [Online]. Available: http://dx.doi.org/10.1108/AEAT-04-20150097

[19] P. Sadegh J. C. Spall, “Optimal random perturbations for multivariable stochastic approximation using a simultaneous perturbation gradient approximation", IEEE Trans. Automatic Control, vol. 43, no. 10, pp. 1480-1484, 1998. [Online]. Available: http://dx.doi.org/10.1109/9.720513

[20] U.S. Military Handbook MIL-HDBK-1797, 19 December 1997. 